\title{
Phronesis
}

\section{Circulation de savoirs entre institution de formation et terrains scolaires : analyse de dispositifs de formation à l'enseignement de la production écrite en Suisse romande Circulation of Knowledge between Teacher Training Institution and School field; Analysis of Teachers Training Devices on the Teaching of Written French}

\section{Roxane Gagnon et Véronique Laurens}

Volume 5, numéro 3-4, 2016

Quels discours pour quel développement professionnel ?

URI : https://id.erudit.org/iderudit/1039087ar

DOI : https://doi.org/10.7202/1039087ar

Aller au sommaire du numéro

Éditeur(s)

Université de Sherbrooke

Champ social éditions

ISSN

1925-4873 (numérique)

Découvrir la revue

Citer cet article

Gagnon, R. \& Laurens, V. (2016). Circulation de savoirs entre institution de formation et terrains scolaires : analyse de dispositifs de formation à

l'enseignement de la production écrite en Suisse romande. Phronesis, 5(3-4),

69-86. https://doi.org/10.7202/1039087ar

\section{Résumé de l'article}

Dans ce texte, nous analysons le traitement de la pratique professionnelle dans deux séquences de formation portant sur l'enseignement de la production écrite. Dans un premier temps, nous observons les caractéristiques des dispositifs de formation prévoyant l'alternance entre le terrain scolaire et l'institution de formation. La focale est ensuite mise sur les interactions entre formateur et formés à l'intérieur de deux activités de formation ciblant le retour sur la pratique. L'intérêt de cette double perspective est de mieux comprendre la circulation des savoirs d'un lieu à un autre et comment cette circulation est mise au service du développement de l'agir enseignant. 
Circulation de savoirs entre institution de formation et terrains scolaires : analyse de dispositifs de formation à l'enseignement de la production écrite en Suisse romande

Roxane GAGNON*, Véronique LAURENS**

*HEP Vaud

UER Didactique du français

Avenue de Cour 33 (bureau C37-128)

Lausanne, Suisse, 1014

Roxane.Gagnon@hepl.ch
**Université Sorbonne Nouvelle-Paris 3

EA2288 DILTEC

Département de didactique du FLE

46 rue Saint-Jacques

Paris, France, 75005

veronique.laurens@univ-paris3.fr

Mots-clés : Formation des enseignants, didactique du français écrit, alternance entre institution de formation et terrain scolaire, double triangulation, circulation des savoirs.

Résumé : Dans ce texte, nous analysons le traitement de la pratique professionnelle dans deux séquences de formation portant sur l'enseignement de la production écrite. Dans un premier temps, nous observons les caractéristiques des dispositifs de formation prévoyant l'alternance entre le terrain scolaire et l'institution de formation. La focale est ensuite mise sur les interactions entre formateur et formés à l'intérieur de deux activités de formation ciblant le retour sur la pratique. L'intérêt de cette double perspective est de mieux comprendre la circulation des savoirs d'un lieu à un autre et comment cette circulation est mise au service du développement de l'agir enseignant.

\section{Title: Circulation of Knowledge between Teacher Training Institution and School field;}

\section{Analysis of Teachers Training Devices on the Teaching of Written French}

Keywords: Teacher Training, Written French Language Instruction, Combined School Field and Training Institution Scheme, Double Triangulation, Circulation of Knowledge

Abstract: In this contribution, we analyse the treatment of professional practice in two training sequences dedicated to the teaching of written French. Firstly, we examine the characteristics of the training devices organised with a combination of school field and training institution scheme. We then focus on interactions between trainer and trainees within two training activities aimed at sharing and reflecting on practicum. The interest of this double perspective lies in the understanding of knowledge circulation between school field and training institution and how this circulation serves the development of teachers' action. 
L'un des principaux objectifs des programmes des institutions de formation est de permettre à l'enseignant d'être progressivement apte à analyser son action professionnelle en situation'. Cette réflexivité vise à former des enseignants, capables d'" agir en tant que professionnel critique et porteur de connaissances et de culture ", " de s'engager dans une démarche individuelle et collective de développement professionnel » et " d'agir de façon éthique et responsable dans l'exercice de ses fonctions $\|^{2}$. À cette fin, les institutions de formation à l'enseignement proposent des dispositifs de formation en alternance favorisant l'articulation entre les compétences professionnelles et les compétences didactiques, requises pour la formation des élèves. La présente étude porte précisément sur les activités de formation mobilisant la pratique professionnelle en milieu scolaire afin de cerner les objets de savoirs mobilisés et les modes de construction de ces objets.

Notre étude fait partie d'un projet plus large ${ }^{3}$ sur les pratiques de formation dans le domaine de la production écrite. Une analyse préliminaire des plans de formation et des descriptifs de cours (Gagnon \& Surian, 2012) nous a menées à constater que l'articulation avec le terrain scolaire était pensée de manière différente selon l'institution de formation et selon la filière d'enseignement à laquelle se destinaient les futurs enseignants. Elle se déroule dans le cadre de stages pour la formation initiale à l'enseignement primaire; pour l'enseignement secondaire, elle est pensée en cours d'emploi. Cette enquête préliminaire fait également ressortir trois principaux thèmes en lien avec la pratique sur le terrain scolaire : la conception de dispositifs didactiques, leur mise en œuvre pendant le stage et le retour sur la pratique conduite.

Deux questions phares orientent notre étude :

1. Comment se caractérisent les dispositifs de formation prévoyant l'alternance entre le terrain scolaire et l'institution de formation? Quels sont les contenus et démarches convoqués?

2. Comment les savoirs circulent-ils entre le contexte de la formation et celui de la pratique sur le terrain scolaire?

Notre analyse porte plus particulièrement sur le traitement de la pratique professionnelle dans deux séquences de formation ${ }^{4}$ (désormais SF; séquence de formation primaire : SFP; séquence de formation secondaire : SFS). D’un point méthodologique, notre démarche suit deux étapes. Nous analysons les activités incluses dans les séquences, observant les objets convoqués et l'enchainement des activités. Puis, nous portons la focale sur les interactions entre formateur et formés à l'intérieur de deux activités de formation ciblant le retour sur la pratique en terrain scolaire, procédant à une analyse des échanges regroupés par thématique.

\section{Repères théoriques pour situer l'alternance terrain-institution dans la formation des enseignants}

Dans cette partie, nous développons les présupposés théoriques qui sous-tendent notre conception du développement de l'adulte, de la formation des enseignants et de l'ingénierie de formation. Ces concepts ont émergé dans un contexte marqué par la volonté de professionnaliser les acteurs du monde de l'enseignement et d'accroitre l'efficacité de la formation. L'énoncé

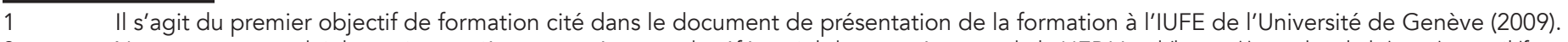

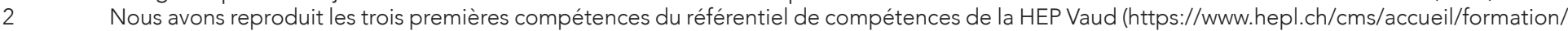
referentiel-de-competences.html).

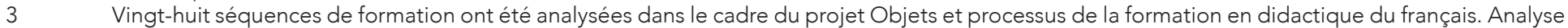

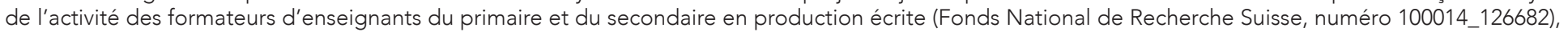

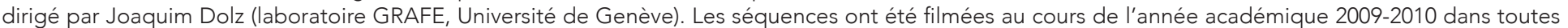

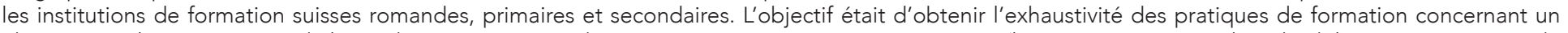

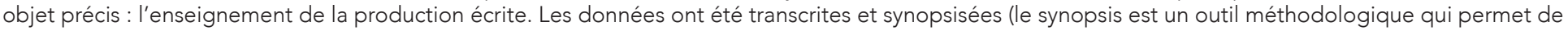

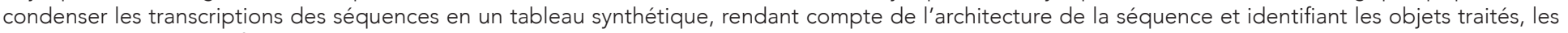
activités, les supports (cf. Schneuwly \& Dolz, 2009)).
}

4 Désormais SF ; pour séquence de formation primaire, nous réduisons à SFP et séquence de formation secondaire devient SFS. 
de notre cadrage théorique est suivi de l'explicitation de notre approche en vue de cerner les allers retours entre institution de formation et terrain professionnel, fruits d'interactions complexes entre plusieurs dimensions.

\subsection{Vers une nouvelle épistémologie professionnelle}

Les travaux s'intéressant à la formation des enseignants mettent en exergue la tension existant entre, d'une part, les exigences et contraintes qui sous-tendent l'action de l'enseignant dans sa pratique et, d'autre part, celles qui caractérisent les activités de production de savoirs scientifiques : "Métier sans savoirs, savoirs sans métier : telle est la contradiction majeure qui peut opposer l'enseignement comme pratique et la recherche sur l'enseignement » (Vanhulle \& Lenoir, 2005, p. 46).

Dans la conjoncture actuelle, les diverses revendications en vue d'une recherche utile pour la formation d'enseignants compétents et professionnels sont autant de tentatives en vue de mieux arrimer théorie et pratique (Tardif, Lessard \& Gauthier, 1998; Bourdoncle \& Lessard, 2003). Pour de nombreux auteurs, I'articulation entre l'action de formation et la recherche sur la formation serait plus bénéfique si l'on sortait d'une logique binaire opposant théorie et pratique (Vanhulle et Lenoir, 2005; Merhan, Ronveaux \& Vanhulle, 2007; Vinatier, 2013).

\subsubsection{Le lien entre formation et pratique dans la construction des savoirs professionnels de l'enseignant}

L'identification des savoirs de la formation, fortement composites, a toujours engendré une série de tensions entre les références multiples nourrissant la construction des savoirs professionnels (Vanhulle, 2009). La combinaison et l'intégration de ces références demeurent un enjeu majeur pour les futurs enseignants, tant sur un plan épistémique, qu'identitaire. Ceci impacte la construction identitaire du professionnel et l'utilisation qui est faite des savoirs académiques dans la profession enseignante (Fillietaz, 2013, p. 295).

La construction de ces savoirs chez l'enseignant en formation est favorisée par le développement d'une attitude de praticien réflexif (Schön, 1994). On comprend alors pourquoi l'analyse de pratiques constitue l'une des caractéristiques-clés de ce modèle de formation, développé depuis les années 1990 (Perrenoud, 1994). Ainsi, un modèle de formation professionnelle à I'enseignement a progressivement été façonné (Tardif \& Ziarko, 1997; Dugal, 2003) en réaction au modèle processus-produit à visée applicationniste (Crahay, 2006) : les savoirs professionnels naissent de la pratique et de l'analyse de la pratique en situation, ils ne se transmettent pas, mais se construisent (Vanhulle, 2009 : 244; Vinatier, 2013). Le lien classique qui pose les savoirs de référence comme précédant le passage à la pratique professionnelle n'est en fait plus vu comme naturel, mais comme artificiel (Vanhulle, 2008). La formation ayant recours à l'alternance médiatise le lien entre objets de savoir et développement de la pratique professionnelle au niveau de l'individu qui se forme à condition que celui-ci soit engagé dans une dynamique qui lui permette de façonner ses savoirs propres, nourris par les différents types de savoirs qu'il mobilise (Vanhulle, Mottiez Lopez \& Deum, 2007).

\subsubsection{Du développement de l'adulte et du professionnel}

Bien que Vygotski ait défendu la thèse selon laquelle l'adulte et l'enfant ne se développent pas de la même manière, il est fait abondamment référence aux thèses vygotskiennes dans les modèles théoriques des travaux en formation professionnelle (Filliettaz, 2013). Le concept de zone de développement proximal y est utilisé en référence au guidage et aux interactions entre novices et experts (Prot \& Schneuwly, 2013). Dans le champ de la didactique professionnelle, Mayen utilise le concept de situation potentielle de développement qu'il définit de la façon suivante «l'ensemble des conditions qu'une situation doit remplir pour engager puis étayer le processus de développement des compétences » (1999, p. 66). II est possible d'utiliser le concept de zone d'efficacité de la formation pour parler de ce que le formé arrive à faire avec le soutien du formateur, dans un dispositif de formation. Selon Bronckart (2008), l'identification d'une telle zone tient toujours du pari. En effet, les apports externes ne sont générateurs de développement que dans la mesure où ils sont traitables par le formé en son état actuel de développement. Pour Clerc-Georgy et Ducrey Monnier (2013), les savoirs scientifiques revêtent une fonction de restructuration de la pensée des étudiants dans la mesure où ils entrent en conflit avec la conception spontanée et l'expérience de la pratique des étudiants.

Pastré (2005) considère d'ailleurs que le développement chez l'adulte est plus historique que génétique et qu'il se traduit par des crises identitaires profondes : le conflit créé par le décalage entre la réalité du processus chez un sujet et la conscience qu'il en a amène le dépassement des anciennes conceptions. Clot parle du développement de l'adulte comme du pouvoir

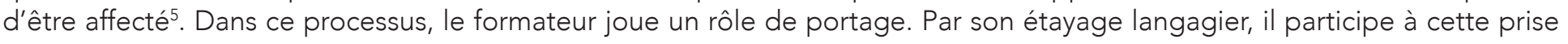

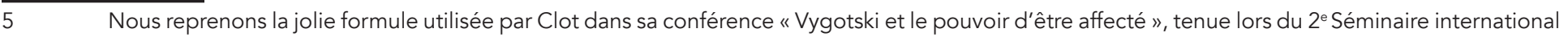


de conscience et à cette prise de distance du formé, en ce qu'il contribue à désimpliciter les savoirs inscrits dans les pratiques professionnelles (Mayen, 2002; Filliettaz, 2013). Cet étayage favorise la réflexivité; laquelle, dans le processus de développement, permet au formé d'étayer sa pratique et de devenir historien de son propre passé (Pastré, 2005).

L'idée de reprise, de réinvestissement, de transformation est fondamentale lorsqu'il s'agit d'essayer de comprendre comment une personne se développe. Bronckart pose comme condition de développement le fait de devenir " apte à réinvestir ses acquis personnels dans le monde social », et ainsi de devenir " apte à contribuer à la transformation des préconstruits collectifs (...) » (2008, p. 211). II ajoute que « cette aptitude de créativité sociale en présuppose une autre, préalable, ayant trait aux conditions d'accès des individus à ces mêmes préconstruits collectifs » (p. 223). Dans le processus, l'individu prend conscience des différentes positions en présence dans le débat social autour de la profession, restructure ses représentations en attribuant de nouvelles significations à ses actes propres et au travail dont ils réfèrent.

\subsubsection{Des conceptions de dispositifs de formation en alternance}

Comment intégrer l'analyse des pratiques à la formation afin que le formé puisse mieux situer/interpréter ces pratiques, et en tirer des règles ou des stratégies d'action? Par quels dispositifs rend-on possible la construction de savoirs professionnels?

Vanhulle, Merhan et Ronveaux recensent quatre modèles de formation en alternance attestés en formation d'enseignants (2007, p.11):

- un modèle de type applicationniste où la théorie précède la pratique,

- un modèle acculturateur qui fonctionne par immersion sur le terrain,

- un modèle intégrateur précoce qui est conçu à partir d'allers-retours entre la formation et le stage par le biais d'analyses,

- un modèle intégrateur par étapes « (...) où la formation théorique est censée soutenir l'entrée progressive dans une pratique guidée puis davantage autonome, la théorie étant susceptible de se laisser infléchir et questionner par des problématisations issues des situations de pratique. »

Ces différents modèles de formation donnent des fonctions spécifiques à l'alternance et déterminent le lien émergeant ou établi entre le lieu de formation et l'espace de la pratique (Bourgeon, 1979, cité dans Vanhulle, Merhan, Ronveaux, 2007, p.11):

- Est-on dans la juxtaposition (espaces indépendants)?

- Dans l'association (formation sur le terrain sans coordination)?

- Ou dans l'intégration (interdépendance entre formation en institution et sur le terrain)?

Ce sont les modèles intégrateurs qui retiennent notre attention, car ils s'inscrivent dans l'épistémologie professionnelle décrite supra. Vanhulle, Mottier, Lopez et Deum donnent quelques caractéristiques de ces dispositifs :

(I) es dispositifs de formation sont censés médiatiser le rapport des futurs enseignants à la profession et aux savoirs qu'elle implique. Entre autres, des démarches réflexives systématiques et récurrentes, organisées tant à l'université que sur les lieux de stages, offrent un espace ouvert à la confrontation bienveillante des expériences et des points de vue (...). Ces démarches tendent à aider les étudiants à transformer des savoirs multiples en significations utiles pour penser leur agir professionnel et leur image d'eux-mêmes dans cet agir (2007, p. 243).

Les institutions de formation investiguées par la recherche se targuent toutes dans les présentations des cursus d'appliquer les principes de cette épistémologie professionnelle. La formation pratique dispensée suit l'un ou l'autre des modèles intégrateurs. L'optique qui est la nôtre ici consiste à analyser les unités de formation et de voir comment ces unités intègrent les savoirs de la pratique.

Vygotski, le 17 octobre 2008, à Genève. 


\subsection{L'alternance en formation : circulation des savoirs entre discours et action}

L'originalité et l'intérêt de notre démarche sont d'examiner les savoirs de la formation et de l'alternance entre théorie et pratique du point de vue des savoirs strictement didactiques, dans un domaine et sur un objet spécifique, la formation à l'enseignement de la production écrite. Cette centration sur le savoir, dans les pratiques en contexte de formation et sur le terrain scolaire, nous distingue des travaux en didactique professionnelle. La réflexivité du formé porte sur des savoirs professionnels en lien à un objet précis.

Deux postulats organisent notre conception de l'alternance : la prise en compte d'une double triangulation didactique ainsi que la circulation des savoirs dans le but d'amener le formé à se situer dans ce double système et d'identifier, et de faire sien dans la pratique, les savoirs reçus en contexte de formation.

\subsubsection{Double triangulation didactique et circulation des savoirs}

D'un point de vue didactique, la formation des enseignants peut être considérée comme un système propre, régi par des phénomènes, des règles et des modes de fonctionnement similaires à ceux qui règlent le système d'enseignement (Portugais, 1995). Sous cet angle, l'analyse des situations de formation des enseignants requiert la prise en compte d'une double relation triadique : le système de la formation articulant les pôles formateur, formé et objet de savoir de la formation et le système d'enseignement formé du triptyque enseignant, élève, objet de savoir enseigné. Pour Portugais, le savoir d'expérience se construit dans l'interaction entre la situation didactique (ou situation du travail enseignant) et la situation de formation : son élaboration est progressive, dépendante des « déséquilibres et rééquilibrations successifs qui sont induits par les contraintes de l'expérience et de l'action » (ibid., p. 340).

Faisant nôtre la notion de circulation des savoirs, développée au départ par Dugal et Léziart en 2004, nous l'utilisons afin de penser les phénomènes de relais des contenus du contexte de la formation à celui de la pratique (Gagnon, 2010). Martinand (2006) soutient que la formation a une influence médiate et différée, car la circulation des savoirs et des compétences dépend d'un régime d'expertise noué en un lieu, à un moment et avec des partenaires définis. Pour rendre les savoirs acceptables et efficaces dans la pratique formatrice, il faut les (re) - problématiser dans le cadre d'une autre pratique (ibid., p. 6). Une circulation des savoirs qui favorise l'exploitation de la double triangulation didactique révèle le savoir-faire visé en formation et permet une transformation de celui-ci en fonction des intérêts du formé, des objectifs d'apprentissage, du contexte, des élèves. Elle est rendue possible par des outils de formation qui comportent une double facette, un usage en formation et un usage dans l'enseignement, et qui, idéalement, incluent une réflexion sur ce double usage. Ces mouvements de circulation de savoirs impliquent la construction d'une posture de secondarisation (Bautier \& Rochex, 2004), à savoir une capacité à ressaisir les objets pour les questionner, les décontextualiser et les reconfigurer.

\subsection{2 Émergence d'une co-construction des savoirs à partir des interactions en formation : entre le faire et le dire sur le faire}

Les activités de formation dédiées au retour des expériences vécues en stage ou en emploi semblent particulièrement propices à l'observation de la circulation des savoirs, car elles donnent lieu à des discussions collectives sur les pratiques, ce qui représente une (première) mise à distance de l'expérience. C'est pourquoi nous avons choisi d'analyser plus finement ces activités de retour d'expériences au niveau des interactions qui se déroulent lors de ces activités dans le temps de formation afin de saisir comment se formalise le faire du formé à partir du dire sur ce faire. Que se passe-t-il dans les interactions de formation entre le formateur et les formés? Quels contenus de savoirs sont-ils évoqués? Y a-t-il transformation ou objectivation des savoirs au gré des échanges?

Analyser des interactions au cours de séances de formation à l'enseignement initial est l'une des spécificités et des originalités de notre étude. Nous prenons appui pour ce faire sur deux grands ensembles de travaux centrés sur l'analyse des interactions didactiques en classe de langue d'une part, et sur les verbalisations des enseignants sur leurs pratiques effectives.

L'orientation actionnelle des recherches sur les discours de la classe de langue est développée en sciences de l'éducation depuis une vingtaine d'années, notamment en didactique des langues (cf. Altet, 2002; Cicurel \& Bigot, 2005; Filliettaz \& Schubauer-Leoni, 2008). Les interactions didactiques sont considérées comme une voie d'accès privilégiée aux pratiques effectives des enseignants telles qu'elles émergent au fil des interactions. L'enseignant est considéré non seulement comme porteur d'un discours et interactant au sein d'une classe mais aussi comme « auteur d'actions » dont le but est de transmettre des contenus de savoir à d'autres (Cicurel, 2005, p. 24). 
Les actions de l'enseignant n'étant qu'en partie interprétables à partir des interactions didactiques, le deuxième ensemble de recherches de référence porte la focale sur les verbalisations des enseignants à propos de leur propre pratique. Ceci permet de tenter d'avoir accès à la dimension cachée de l'agir enseignant (la genèse de l'action, les motifs, l'intentionnalité, la prise de décisions) (Cicurel, 2011, p. 240). L'agir enseignant est ainsi vu comme constitué « [...] par la somme des actions, des intentions, des émotions et des projets de l'enseignant » (ibid., p. 239). Dans le cadre de la formation d'enseignants, différents dispositifs sont utilisés pour susciter la réflexivité des étudiants sur leur agir professionnel en construction : analyses collectives des pratiques de stages, écrits réflexifs, portfolios, dossiers de développement professionnel (Vanhulle, 2009, p. 245).

\subsubsection{Sept modalités pour caractériser la circulation des savoirs entre formation et pratique}

De manière à déterminer comment le système de formation et le système didactique s'articulent dans les dispositifs de formation, nous employons le terme de modalité. Les modalités renvoient à des degrés variables d'actualisation de l'agir enseignant. A partir de ce qui ressort du cadre théorique posé, nous proposons sept modalités permettant de qualifier la circulation des savoirs dans les dispositifs d'alternance:

1. la proportion des blocs d'activités consacrés aux savoirs pratiques dans la séquence;

2. la place de ces blocs dans la séquence : surviennent-ils au début, au milieu ou en fin de séquence? ;

3. les rapports établis entre les expériences antérieures du formé, le vécu du terrain ou les contenus et démarches de formation;

4. I'exhaustivité de l'objet de formation devant être mis en pratique : dans quelle mesure l'activité pratiquée sur le terrain est-elle représentative de l'activité professionnelle au quotidien, pratique-t-on un aspect pointu du métier ou le métier dans son ensemble? ;

5. la modélisation de la pratique à mettre en œuvre sur le terrain, son exemplification par homologie;

6. I'explicitation : la place de la réflexion individuelle, de la reformulation individuelle de la pratique; la place de l'étayage du formateur dans l'explicitation de la pratique; la place des supports de formation dans l'accompagnement de l'explicitation de la pratique;

7. la présence de conflits entre les concepts quotidiens des formés, l'expérience pratique et les savoirs de la formation.

\section{Articulation du système de formation et du système didactique dans deux SF emblématiques}

Les SF proposant aux étudiants en formation initiale à l'enseignement primaire de faire, à l'intérieur d'un cours de didactique du français, un stage pratique sur le terrain scolaire constituent le point de départ de notre réflexion sur la question de la présence et de la place de l'alternance entre le terrain et l'institution de formation dans le corpus recueilli. Cette première focalisation a pour propos d'observer l'impact potentiel réciproque entre les situations vécues en formation et les situations vécues en stage pour le développement professionnel des étudiants futurs enseignants.

Huit SF sur les 28 enregistrées, transcrites et mises en synopsis, comportent des temps de terrain. Une distinction à rappeler, dont nous avons pris compte dans notre analyse : dans notre corpus, l'expérience du terrain s'illustre de deux manières, selon que les étudiants se destinent à l'enseignement primaire ou à l'enseignement secondaire. Pour le primaire, les étudiants vivent de premières expériences de terrain scolaire sous forme de stages; pour le secondaire, les étudiants en formation sont en emploi comme enseignants, le terrain est donc pour eux une expérience dans le quotidien de leur métier, et c'est au fil de certaines activités de formation que l'on trouve des traces du lien avec le terrain éprouvé dans le cadre professionnel. Six séquences relèvent de la formation à l'enseignement primaire, deux séquences font partie des formations à l'enseignement secondaire.

De manière à décrire le fonctionnement des dispositifs en alternance, nous détaillons les lieux et les formes d'alternance de deux séquences emblématiques, l'une en formation à l'enseignement primaire, l'autre en formation à l'enseignement secondaire. Nous décrivons, au sein de chaque séquence, l'enchainement des activités portant sur les savoirs issus de la pratique. L'expérimentation des contenus de savoir issus de la pratique a-t-elle lieu au début de la séquence, survient-elle à la fin de celle-ci? La séquence intègre-t-elle un moment de retour réflexif sur cette expérimentation pratique? II s'agit d'examiner ensuite le type de pratique convoquée et le rapport que celle-ci institue entre le formé et son action : sommes-nous en présence 
d'une pratique simulée, en contexte réel de stage, en responsabilité, etc. ? Nous pointons les objets en lien à l'enseignement de la production écrite convoqués et, s'il y a lieu, détaillons les outils au service de l'articulation terrain et formation.

\subsection{La SF à l'enseignement primaire : un projet de communication comme outil de planification et de retour sur la pratique}

La séquence «Enseignement de la production écrite au cycle 2 » compte deux blocs d'activités sur la pratique sur le terrain scolaire. Le premier bloc survient en milieu de séquence : les étudiants, en $3^{3}$ année de formation à l'enseignement primaire, sont invités à préparer, réaliser et analyser des projets d'écriture en stage. Le formateur leur montre en début de séquence des projets de communication réalisés par des formés des années antérieures. En groupe, les étudiants font le choix d'un genre textuel selon la démarche "S'exprimer en français $»^{6}$ en vue de leur stage. Le formateur propose deux entrées possibles pour la réalisation d'un projet d'écriture : le genre ou la situation de communication. II fournit un canevas en vue de la réalisation de la séquence ou du projet en stage.

Le deuxième bloc survient à la fin de la séquence. C'est le moment où le projet de communication autour du genre est réalisé par le stagiaire avec ses élèves. Le temps de terrain est suivi d'un retour réflexif sur l'expérience réalisée en stage. Les étudiants sont amenés à échanger en groupes autour des réussites et les difficultés rencontrées lors du stage. Les étudiants analysent une des difficultés vécues pendant le stage en fonction des trois pôles du triangle didactique pour envisager des pistes d'actions. En plus de cette analyse de difficultés, le formateur demande aux groupes d'étudiants de concevoir deux panneaux: le premier articule des points théoriques vus dans le cours et les aspects plus pratiques rencontrés sur le terrain; le second présente une synthèse des réflexions des étudiants au sein de chaque groupe. Dans cette séquence, le projet sert de liant entre les contenus et les contextes de la formation et de la classe. Les concepts vus dans la formation font l'objet de reprises, de transformations et, grâce au retour réflexif effectué par l'entremise des panneaux, les étudiants peuvent faire dialoguer les expériences sur le terrain et les concepts scientifiques en lien au projet de communication.
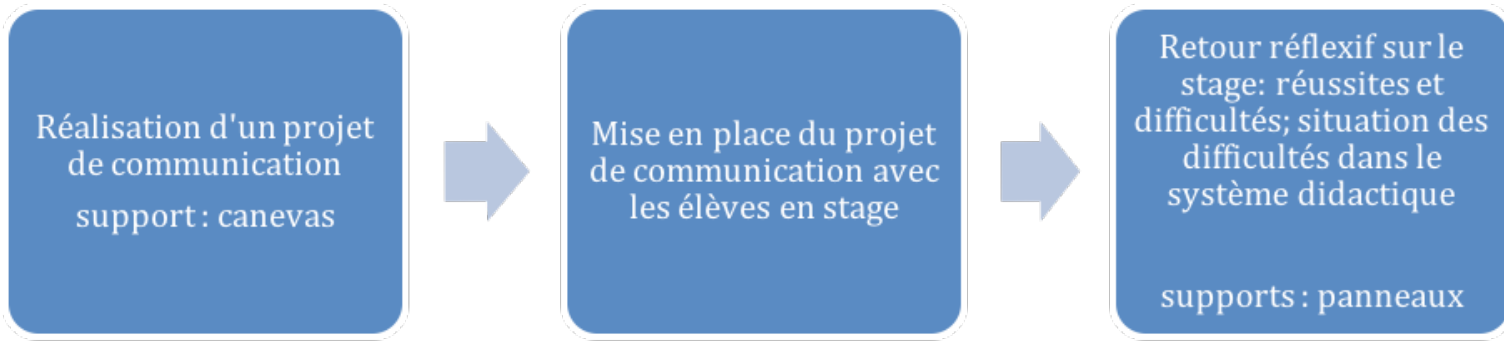

Figure 1 : Modèle d'alternance SFP-04. Le projet de communication secondarisé.

\subsection{La SF en enseignement secondaire : quand la mise en pratique fait débattre de l'utilité du dispo- sitif présenté}

Dans cette séquence, le formateur présente l'histoire de l'enseignement de la littérature et expose comment la littérature est devenue objet d'enseignement. II situe trois poèmes par rapport à leur régime de littérarité et propose aux étudiants, en $2^{e}$ année de formation à l'enseignement au secondaire, de concevoir des activités d'enseignement pour travailler le régime de littérarité à partir de poèmes que les enseignants formés ont apportés. Le 2e bloc de la séquence porte sur l'élaboration de séquences didactiques pour travailler la poésie. La dernière partie de la séquence comporte des activités sur l'exploitation en classe de contenus de formation : les étudiants commentent la mise en œuvre des activités sur la poésie inspirées de ce qui a été vu en formation.

Dans ce dispositif, il est possible de parler des « risques de l'expérience » (Mayen, 2007, p. 93) : « la confrontation aux expérimentés en situation peut être celle du dénigrement de la formation, de son utilité et de ses apports ». Dans notre cas précis, l'expérience pratique mobilisée par le formé entraine une sous-estimation de la nécessité d'apprendre et de mettre en œuvre une séquence d'enseignement sur la poésie, telle qu'elle est conçue dans la formation. Ici, les manières d'agir et de raisonner la pratique ne semblent pas assez constituées et appropriées pour que les formés résistent " aux risques du terrain » (Mayen, 2007, p. 94). Dans la partie suivante, nous revenons plus en détail sur cette séquence.

6 Dolz, J., Noverraz, M. \& Schneuwly, B. (2001). Séquences didactiques pour l'oral et pour l'écrit. Bruxelles : De Boeck. L'ouvrage est l'un des principaux moyens d'enseignement officiels pour le $2^{\mathrm{e}}$ cycle du primaire. 

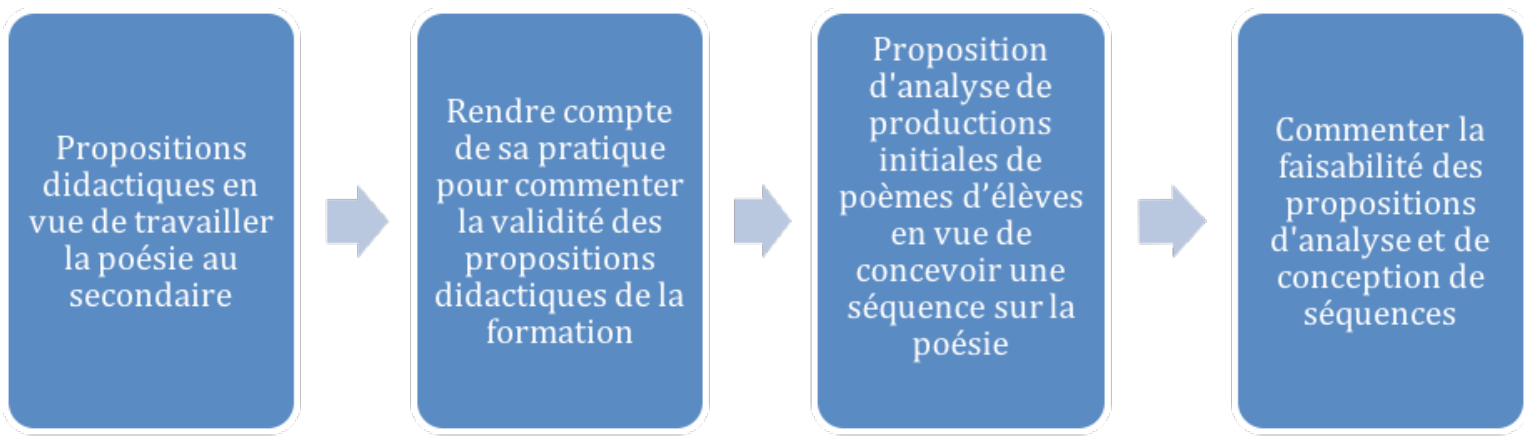

Figure 2 : Modèle d'alternance SFS-08. Rendre compte de sa pratique pour mieux débattre des contenus et démarches vus en formation.

\subsection{Diverses modalités de circulation des savoirs dans les dispositifs d'alternance}

Peut-on dire que dans ces séquences la pratique est secondarisée? Quels savoirs sont rendus conscients et opératoires pour les formés? Dans quelle mesure les dispositifs d'alternance favorisent-ils la construction d'une posture de professionnel de l'enseignant? De manière à synthétiser les modèles d'alternance recensés, nous situons ceux-ci au regard des sept modalités présentées supra (cf. partie 1.2.1.).

Le premier indicateur que nous pouvons observer pour tenter de répondre à ces questions concerne la proportion des blocs savoirs pratiques dans la séquence. La séquence SFP-04 contient 16 activités sur 49 (33 \%) consacrées à la pratique, comparativement à 2 sur 18 activités (11\%) dans la séquence SFS-08.

Sur la place des blocs pratiques dans la séquence, de telles activités surviennent dans la SFS en réaction à ce qui est présenté en formation. C'est dans l'après-coup de la mise en pratique que les frictions entre la réalité du terrain et ce qui est proposé en formation surviennent. Le rapport à une pratique antérieure du formé se fait par le biais de l'objet " poème » que le formé doit apporter en formation. Dans la SFP, les rapports établis entre l'expérience du terrain et entre les contenus et démarches de formation se font par l'entremise d'une analyse de projets antérieurs d'étudiants. Un peu plus loin dans la séquence, le formateur demande aux étudiants de se remémorer des expériences antérieures de stage dans lesquelles ils ont mobilisé le travail de lecture en compréhension pour nourrir la production textuelle des élèves. En fin de séquence, le retour sur le temps de terrain donne la possibilité aux étudiants de s'exprimer sur les obstacles et succès rencontrés en stage. Le rapport à une pratique professionnelle se construit à divers moments de la séquence et de diverses manières.

Par rapport à l'exhaustivité de l'objet de formation devant être mis en pratique, la SFP s'intéresse à la mise en place d'un projet de communication ou à une séquence sur un genre textuel. Ces deux objets sont en lien direct avec l'une des trois grandes finalités de la discipline, la production-compréhension de textes, stipulées dans les documents officiels. Pour le secondaire, la poésie constitue une des pratiques littéraires privilégiées, la nouveauté apportée par cette séquence est le fait de proposer la création d'une d'ingénierie et non pas d'utiliser des activités existantes tirées de moyens existants.

Dans quelle mesure la pratique demandée fait-elle l'objet d'une modélisation par le formateur? Pour la SFP, le formateur montre la pratique attendue par l'entremise des projets antérieurs des étudiants, ceux-ci exemplifient les attendus du stage et de l'unité de formation, et par l'apport d'un canevas. Pour la SFS, le formateur donne un exemple de séquence didactique à partir d'un poème de Ronsard puis guide les enseignants de manière à ce qu'ils créent eux-mêmes leur propre matériel. Le formateur présente un dispositif didactique, sans intégrer une réflexion sur la mise en pratique concrète de cette ingénierie auprès des élèves.

L'explicitation de la pratique se fait de façon fort différente dans les deux séquences. Les étudiants en formation à l'enseignement primaire reformulent collectivement et individuellement leur pratique par des activités formalisées de discussion collective, s'appuyant sur des supports (ils doivent créer un panneau qui fait la synthèse des expériences vécues en stage et un autre, qui fait la synthèse des concepts de formation mobilisés lors du terrain). Dans le cas de la SFS, le retour sur la pratique n'est pas formalisé, secondarisé, par des supports. II n'est pas non plus planifié, puisque les activités de retour sur la pratique

\footnotetext{
$7 \quad$ Quatre critères nous permettent d'identifier une activité dans le cours d'une séance de formation : 1) la présence d'une consigne et d'une tâche (ce que l'élève doit faire), 2) l'apparition ou le changement de support ou toute modification de l'environnement matériel, 3) une nouvelle forme sociale de travail (travail en groupe, exposé magistral, etc.) et 4) la présence d'un nouvel objet de formation.
} 
surviennent lors d'interventions de formés, qui viennent bouleverser le déroulement de la séquence prévu par le formateur. L'explicitation de la pratique est à la charge du formé, qui la fait à l'aide de ses propres conceptions, de ses propres moyens.

Les deux séquences se caractérisent par la présence de conflits, mais ceux-ci sont de deux ordres différents. Dans la SFP, les conflits mis en exergue sont les tensions survenant entre les contenus et démarches de formation et entre l'expérience pratique. Dans la SFS, ils portent sur le décalage entre les pratiques et conceptions antérieures des enseignants, qui détiennent déjà une pratique, et l'ingénierie proposée en formation. Rendre les savoirs issus de la formation acceptables et efficaces dans la pratique enseignante demanderait ici de faire davantage écho à l'expertise détenue, de proposer des modèles de pratique qui entrent en dialogue avec les pratiques existantes des formés (Martinand, 2006).

\section{Analyses des activités de retour de terrain dans les deux séquences}

Dans cette section, nous utilisons un grain d'analyse plus fin, en observant les interactions didactiques et la structuration des échanges au cours de deux activités emblématiques. En effet, nous avons fait le choix de nous centrer sur les activités impliquant un retour sur les activités menées sur le terrain, car elles constituent des moments clés pour saisir les interactions entre savoirs issus du terrain et savoirs issus de la formation, au cœur des phénomènes de circulation des savoirs. Dans quelle mesure, ces activités impliquent-elles une intériorisation progressive de démarches d'explicitation, de justification ou d'interprétation pour mener à l'adoption d'une pratique réflexive par l'enseignant formé? Comment, dans et par le discours, les formateurs favorisent-ils le développement professionnel? Afin d'esquisser des pistes de réponse à ces questions, nous effectuons une analyse du contenu et du discours pour dégager:

- Le contexte, au travers de l'explicitation de la consigne permettant de préciser le mode d'interaction entre formateur et formés, le but de l'activité et donc le cadre des échanges;

- Les thématiques qui structurent les échanges à l'intérieur de chacune des activités, l'échange est l'unité dialogale de base entre deux interlocuteurs, il est considéré comme un constituant binaire ou ternaire dans la progression conversationnelle (Sinclair et Coulthard, 1975; Kerbrat-Orecchioni, 1990);

- Les reformulations opérées par le formateur et par les formés, avec une focale particulière sur les reformulations non paraphrastiques, qui opèrent un changement de perspective énonciative (Rossari, 1994), c'est-à-dire toute altération, correction ou modulation du déjà-dit. Dans le discours, les reformulations se matérialisent par des relations grammaticales, lexicales et sémantiques. La reformulation intervient souvent dans les échanges didactiques en raison de son caractère facilitateur; par elle, le locuteur fournit aux destinataires des éléments permettant d'accéder avec une moindre difficulté au sens visé. Elle rend compte de la construction du sens.

Deux activités de retour sur la pratique tirées de deux SF sont analysées. Pour la SFP, il s'agit de l'activité intitulée Évoquer des difficultés rencontrées durant le stage et recenser des pistes d'actions possibles pour y remédier. Pour la SFS, il s'agit de l'activité intitulée Commenter la mise en place des activités d'une séquence sur la poésie.

\section{1 Évoquer des difficultés rencontrées durant le stage au $2^{\mathrm{e}}$ cycle du primaire et recenser des pistes d'actions possibles pour y remédier}

L'activité est précédée par un échange mené par le formateur à propos des satisfactions et des difficultés ressenties et vécues par les étudiants lors du stage. Rappelons que lors de ce stage, les étudiants doivent mettre en place un projet de communication sur un genre textuel avec des élèves du 2e cycle du primaire. Pour organiser l'échange, le formateur projette une liste de thématiques possibles à aborder (situation de communication, objectifs, activités langagières, étapes du projet, place de l'évaluation, gestion du brouillon à l'écrit). Le formateur demande d'abord aux étudiants, en groupe, de lister les difficultés par écrit. Ensuite, un par un, les étudiants évoquent une satisfaction. L'activité qui nous intéresse consiste en l'évocation des difficultés rencontrées durant le stage et à la recension des pistes d'actions pour y remédier. Le cadre des interactions est donc celui d'une invitation à l'échange : d'abord en petits puis en grand groupe. Le formateur pilote les échanges, son objectif est clair: amener les étudiants à formuler des pistes de solution, des stratégies pour dépasser les obstacles.

Le découpage du verbatim de l'activité en fonction des échanges nous permet de dégager huit sous-thématiques : les contraintes de temps, la gestion des contraintes de temps, la difficulté de ne pas avoir terminé la séquence prévue à la fin du stage, la gestion d'un thème imposé par le formateur de terrain, l'organisation de la séquence didactique pour pouvoir évaluer 
ce qui a été enseigné dans un temps court, la mise en place d'une approche intégrée de la langue, l'importance de faire des choix.

L'échange 3, portant sur la difficulté de ne pas avoir terminé la séquence prévue à la fin du stage, est révélateur des orientations du pilotage des échanges par le formateur:

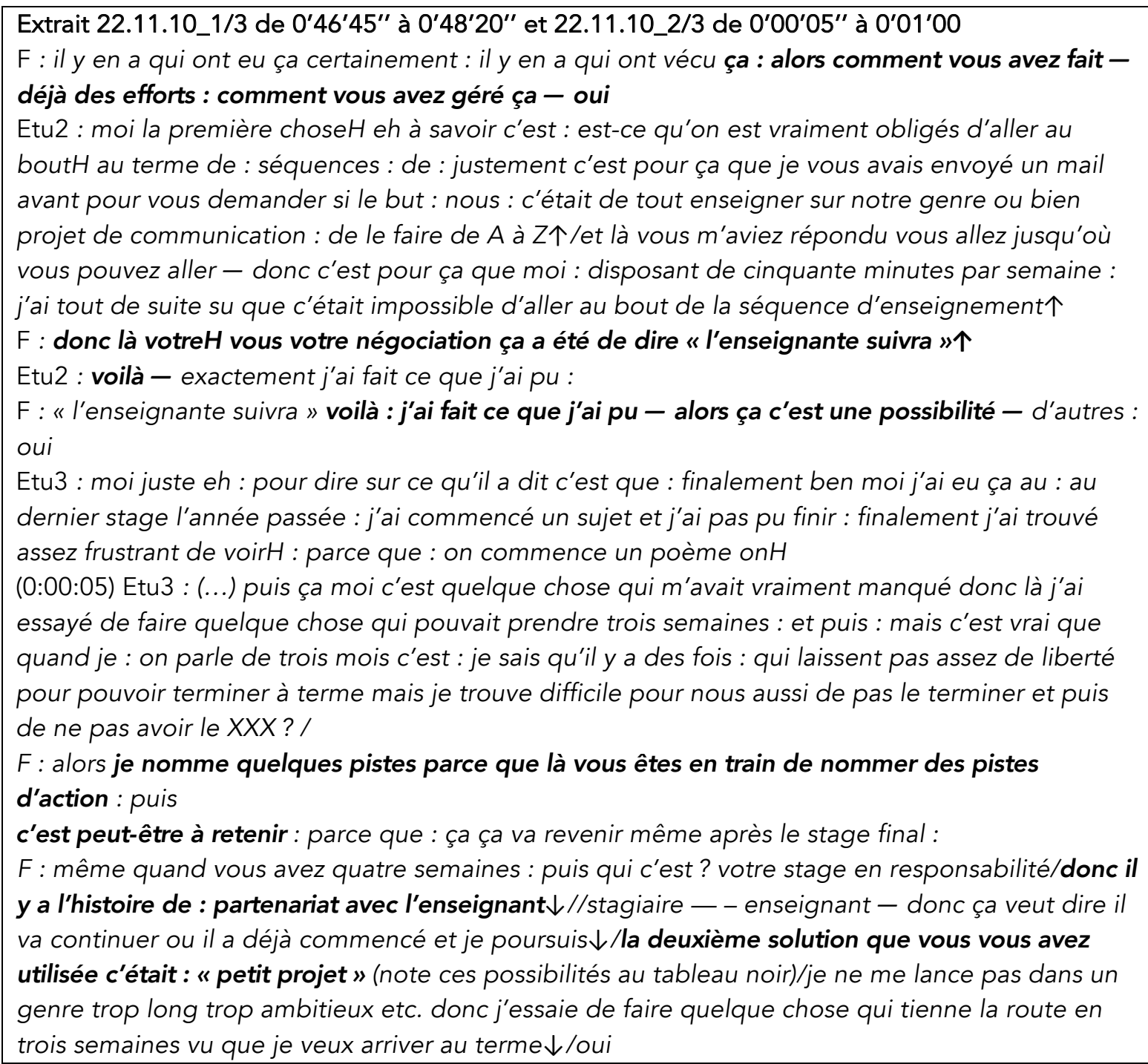

* Les lettres renvoient au formateur (F) et aux étudiants (Etu). Les conventions de transcription sont les suivantes : / // /// : pauses de durée variable ; demanH : unité inachevée ; ${ }^{-}$: intonations montante et descendante ; (entre parenthèses) : commentaires du transcripteur.

Le formateur interpelle les étudiants : « comment vous avez fait», " comment vous avez géré ça ». II cherche à ce que l'échange passe du récit des difficultés aux pistes de solution : le «j'ai tout de suite su que c'était impossible d'aller au bout de la séquence d'enseignement » de l'étudiant est repris par le formateur par " donc là vous votre négociation ça été de dire l'enseignante suivra ». II a recours à une reformulation biaisée, déplaçant le thème pour réorienter l'échange vers la positive. Le formateur veut amener les étudiants à être proactifs : "Alors je nomme quelques pistes parce que là vous êtes en train de nommer des pistes d'action : puis c'est peut-être à retenir : parce que : ça ça va revenir même après le stage final ». II choisit d'infléchir le cours de l'échange en l'orientant clairement du côté des pistes d'actions et d'y impliquer les étudiants alors même que ceux-ci n'ont pas vraiment commencé à en formuler.

L'échange 7 portant sur la mise en place d'une approche intégrée de la langue est celui qui fait participer le plus d'étudiants. 
Le formateur conserve le même objectif : amener les étudiants à formuler leurs difficultés sous forme de pistes d'actions, mais cette fois, en plus d'un déplacement vers le positif et le proactif, les reformulations visent à expliciter aux étudiants ce qu'est une approche intégrée de la langue.

L'étudiant répond à l'appel de piste d'action en évoquant une tension liée au fait que son enseignante formatrice travaille " des gros blocs ». La reprise du formateur " donc là vous avez ciblé sur » vise à amener l'étudiant à expliciter sa pratique davantage : de " gros blocs » vers une focalisation sur la production lecture par le travail du récit d'aventures. L'étudiant répond " voilà », car il maitrise ou il a compris la formulation du formateur aide à comprendre les volets de la discipline mis en exergue par le travail du genre textuel.

L'échange se poursuit, laissant voir les conceptions de la discipline français des étudiants:

\section{Extrait 22.11.10_2/3 de 0'04'00' à 0'05'15}

Etu4 : enfin c'est ça que j'ai remarqué c'est que même si on va pas faire de la grammaire ou du vocabulaire : ou de la conjugaison ça rentre dedans : et puis c'est aussi une sorte de motivation pour les enfants parce qu'ils se rendent même pas compte qu'ils sont en train de faire du français : qu'ils sont en train de $X X$ : puis après ce sera peut-être pas un cours de français proprement parlé mais ça ce sera plus une application donc : préalablement c'est obligatoire de faire un cours de grammaire : pour qu'ils aient intégré certaines notions : puis après je pense que c'est une bonne manière de : d'intégrer ça://

$\mathrm{F}:$ (note)/ok oui :

Etu1 : moi j'ai fait un peu comme Etu c'est un peu auréolé de : les intégrer subtilement : XXX : je les ai intégrés carrément : c'est-à-dire que pendant la situation écrite on avait des ateliers de : grammaire de orthographe : mais c'était : un peu dur et puis c'était : -en même temps - -

F : oui mais ciblé sur l'aide à l'écriture : c'est pas n'importe quoi en grammaire en orthographe c'était vraiment articulé : c'était en articulation avec votre projet : donc ça répond à ça $-:$ ça joue $\uparrow /$

Etu1 : oui

\section{F : c'est utilisation de la structure au senvice de la production : oui}

La question du travail langue-texte dans le cadre d'un projet fait réagir les étudiants, qui participent activement. L'échange n'est plus dual, mais polylogal. Etu 4 conçoit la discipline scolaire de manière assez classique "parce qu'ils ne se rendent pas compte qu'ils sont en train de faire du français ", " puis après ce sera peut-être pas un cours de français à proprement parler », "préalablement c'est obligatoire de faire un cours de grammaire, pour qu'ils aient intégré certaines notions ». La vision partagée va à l'encontre des principes didactiques travaillés dans la séquence : pour Etu4, il faut d'abord exercer la grammaire avant de faire la production; alors que les contenus prônés dans le cours incitent à une articulation réfléchie entre grammaire et texte. Etu1 propose d'intégrer les ateliers de grammaire et d'orthographe à la séquence sur le genre. Le formateur, soucieux de laisser parler les étudiants, réagit rapidement en reprenant les propos des étudiants : " oui mais ciblé sur l'aide à l'écriture, c'est pas n'importe quoi en grammaire en orthographe », qu'il reformule par " c'était en articulation avec votre projet », puis par « c'est l'utilisation de la structure au service de la production ». I| reformule de plusieurs manières l'idée d'articuler langue et projet.

Plus loin dans cet échange, le formateur profite d'une intervention d'un étudiant pour rendre explicites les notions grammaticales pouvant être travaillées à l'intérieur d'une séquence sur un genre textuel : 


\section{Extrait 22.11.10_2/3 de 0'06'45"'à 0'08'00}

Etu5 : (...) alors je les ai fait partir de leurs textes juste pour expliquer qu'il faut mettre des points et : comme il faut : tout ça : alors pour eux c'était plus motivant de travailler la grammaire en somme? /

F : oui : alors là c'est encore autre chose pour moi ça veut dire que : au niveau du programme : on réfléchit pour qu'il y ait un lien entre ce qu'on va travailler : programme : lien entre le genre travaillé et puis les contenus notionnels - (F note cette phrase au tableau noir)/

Etu5 : j'y avais pas forcément réfléchi mais c'était tombé : pile au bon moment et puis :

$\mathrm{F}:$ mais en temps qu'enseignant on peut se dire ça : on peut vraiment se dire : bien tiens : je vais travailler le récit d'aventure : c'est le moment où je travaille par exemple le présent et je les oblige à utiliser le présent de narration : ou je travaille les temps du passé et j'ai travaillé ou je vais travailler sur la partie du passé simple par exemple — d'accord : donc là c'est aussi : ça peut être aussi une possibilitéXXX (F écrit les mots " programme - liens... » au tableau en bas) : autre chose encore $\uparrow$

Le formateur reprend la remarque de Etu 5 « alors je les ai fait partir de leur texte » pour aborder la question du respect du programme : « oui : alors là c'est encore autre chose pour moi ça veut dire que : au niveau du programme (...) on réfléchit pour qu'il y ait un lien entre le genre travaillé et puis les contenus notionnels ». La reformulation amène le développement de l'assertion "partir du texte pour... ». Par l'utilisation du pronom « on », le formateur se place au même titre que l'étudiant, il ajoute d'ailleurs « mais en temps qu'enseignant on peut se dire ça : on peut vraiment se dire : bien tiens : je vais travailler le récit d'aventure : c'est le moment où je travaille par exemple le présent et je les oblige à utiliser le présent de narration ».

Par les reformulations, le formateur oriente l'échange et fait dire aux étudiants ce qu'il compte institutionnaliser : la nécessité de faire des choix quand on enseigne ou, autrement dit, de faire avec les contraintes du terrain; la nécessité de penser l'articulation entre travail de la langue et production textuelle. Par des déplacements de focalisation, les tensions entre terrain et formation sont contournées, oubliées. Les étudiants disent ne pas avoir pu travailler l'entièreté de la discipline — n'ayant pas pu faire de grammaire - ou avoir eu l'impression de lasser les élèves avec ce travail constant sur un genre textuel, le formateur déplace leur attention sur les articulations à penser entre structuration et expression. C'est l'image d'un formateur proactif, stratégique qui ressort.

\subsection{Commenter la mise en place des activités d'une séquence sur la poésie à l'école secondaire}

L'activité sélectionnée pour l'analyse de la SFS sur la poésie survient un mois plus tard, après le retour des vacances de fin d'année. Les enseignants formés ont remis des pistes d'activités au formateur pour travailler un poème dans leur classe. Le formateur démarre par un retour sur ces activités. Alors qu'il effectue cette synthèse, des remarques d'enseignants-formés amènent des échanges autour de la mise en œuvre concrète des activités planifiées auparavant. Ici, comparativement, à l'activité de formation au primaire, le pilotage de l'échange n'est pas à la charge du formateur, mais à celle de l'étudiant qui provoque, quelque part, cette interruption dans la synthèse amorcée par le formateur. L'activité de retour sur la pratique fait moins l'objet d'un contrôle et d'une modélisation par le formateur, en raison de sa méconnaissance des activités réalisées par les formés sur le terrain.

L'extrait suivant illustre le démarrage de l'activité : 


\section{Extrait 14.01.2011_1/4, 0'07'28 à 0'09'30}

$\mathrm{F}$ : ensuite euh troisième euh exercice euh ce serait euh ça aurait été ou ce pourrait être d'établir le rapport de sens entre le premier et le dernier vers - /le premier vers étant sur le chemin de la mort ensuite elle fut prise dans l'Opaque le dernier —/des exercices imaginés le quatrième écrire entre quatre et huit vers libres sur le thème de la mort -/euh simplement ça ça va peut-être un p'tit peu vite on pourrait se demander avant ça comment introduire la notion de vers libre _/si on veut faire écrire des vers libres euh il suffit peut-être pas de leur demander d'écrire des vers libres

Etu : moi j'ai été surpris

$\mathrm{F}$ : ouais $\uparrow$

Etu : parce que

F : savaient tout seuls $\uparrow$

Etu : euh ils sont assez au clair sur ce que c'est la poésie la plupart —/et certains m'ont fait des vers libres $\downarrow$

F : bien —/ils sont assez au clair sur ce qu'est la poésie c'est-à-dire —/c'est quoi la poésie pour eux个

Etu : disons de la forme -ly en a qui ont levé la main en disant est-ce qu'il faut qu'on fasse des rimes est-ce qu'il faut qu'on fasse des euh/déjà qu'ils me posent cette question pis qu'ils fassent le choix de/XXX par facilité

$\mathrm{F}$ : hum hum —/de euh

Etu : qu'ils fassent pas de rime et pas de comptage de vers quoi

$F$ : ouais tout à fait $\downarrow$

Etu : mais ils m'ont posé la question donc ils étaient au courant que ça se faisait comme ça et certains ont choisi de ne pas le faire $\downarrow$

$\mathrm{F}$ : et ils étaient au courant que la poésie pouvait ne pas suivre euh/des normes euh de versification

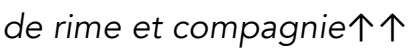

Etu : ben apparemment la poésie scolaire pour eux doit suivre ce genre de choses - parce que

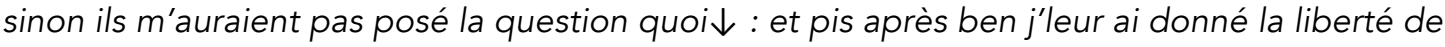
euh de ne pas le faire $\downarrow$ 


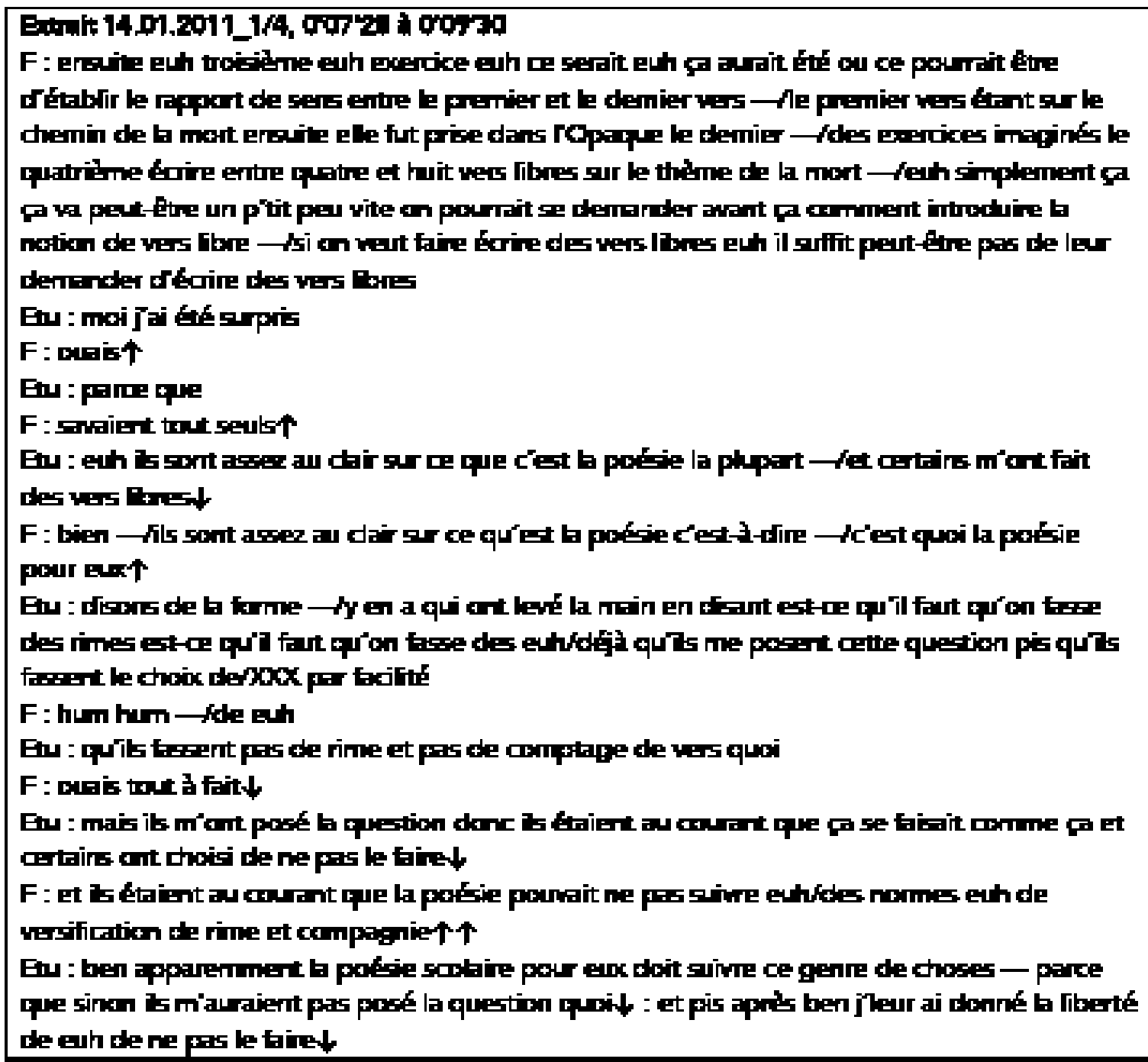

Le premier échange de cette activité démarre donc à partir de l'intervention de l'étudiant qui interrompt le formateur dans sa synthèse et amène une activité dans laquelle un étudiant commente la mise en place d'activités vues en formation dans sa classe. Il porte sur les connaissances des élèves sur la présence ou non des conventions formelles de la poésie.

L'échange 2 aborde la thématique des ateliers de création de poèmes et, encore une fois, c'est un étudiant qui pilote l'échange. Alors que le formateur reprend sa synthèse des activités possibles à mettre en œuvre (faite à partir des propositions reçues), un étudiant l'interrompt en rappelant la possibilité d'organiser des ateliers dans lesquels les élèves rédigent des poèmes à plusieurs. Avec un autre étudiant, il explicite la forme sociale de l'atelier. À la fin de l'échange, le formateur reformule la proposition des étudiants comme une illustration d'une activité qui « pass(e) effectivement (...) à l'intersubjectif ».

L'échange 3 aborde la question du comment lier la textualité et le travail du sens dans les activités. Le formateur, ayant repris sa synthèse des activités possibles, interroge un enseignant formé sur la mise en pratique d'une production initiale d'un poème : 


\section{Extrait 14.01.2011_1/4, 0'07'28 à 0'09'30}

$\mathrm{F}$ : ensuite euh troisième euh exercice euh ce serait euh ça aurait été ou ce pourrait être d'établir le rapport de sens entre le premier et le dernier vers - /le premier vers étant sur le chemin de la mort ensuite elle fut prise dans l'Opaque le dernier —/des exercices imaginés le quatrième écrire entre quatre et huit vers libres sur le thème de la mort -/euh simplement ça ça va peut-être un p'tit peu vite on pourrait se demander avant ça comment introduire la notion de vers libre $-/ s i$ on veut faire écrire des vers libres euh il suffit peut-être pas de leur demander d'écrire des vers libres

Etu : moi j'ai été surpris

$\mathrm{F}$ : ouais $\uparrow$

Etu : parce que

F : savaient tout seuls $\uparrow$

Etu : euh ils sont assez au clair sur ce que c'est la poésie la plupart - let certains m'ont fait des vers libres $\downarrow$

F : bien —/ils sont assez au clair sur ce qu'est la poésie c'est-à-dire —/c'est quoi la poésie pour eux个

Etu : disons de la forme - /y en a qui ont levé la main en disant est-ce qu'il faut qu'on fasse des rimes est-ce qu'il faut qu'on fasse des euh/déjà qu'ils me posent cette question pis qu'ils fassent le choix de/XXX par facilité

$\mathrm{F}$ : hum hum —/de euh

Etu : qu'ils fassent pas de rime et pas de comptage de vers quoi

$\mathrm{F}$ : ouais tout à fait $\downarrow$

Etu : mais ils m'ont posé la question donc ils étaient au courant que ça se faisait comme ça et certains ont choisi de ne pas le faire $\downarrow$

$\mathrm{F}$ : et ils étaient au courant que la poésie pouvait ne pas suivre euh/des normes euh de versification

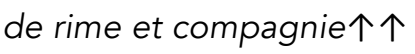

Etu : ben apparemment la poésie scolaire pour eux doit suivre ce genre de choses - parce que

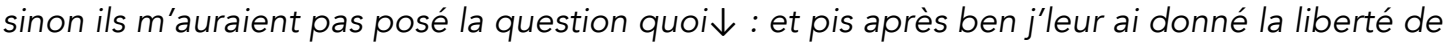
euh de ne pas le faire $\downarrow$

Nous avons évoqué plus haut (cf. partie II) la question des risques de l'expérience (Mayen, 2007), c'est-à-dire le fait que la confrontation au terrain puisse amener le dénigrement de la formation, de son utilité et de ses apports. C'est un peu ce qui se passe dans cet échange, malgré la bonne humeur et la cordialité des échanges entre formateur et enseignant formé : " je n'ai rien fait », « ils ont adoré ». Les contenus de l'échange ne font pas l'objet d'une reformulation, les savoirs ne circulent pas. Le formateur accepte la proposition « d'accord très bien » et oriente rapidement l'échange vers une nouvelle thématique : le traitement du statut référentiel du poème.

La comparaison de ces deux activités de retour sur la pratique montre une construction de l'objet de formation fort différente. Dans l'activité de la SFP, la forme sociale des échanges est clairement définie, le formateur agit comme sur-énonciateur (Rabatel, 2004), il reformule et explicite à son compte — au compte de ce qu'il juge être digne d'intérêt pour le groupe — les propos des formés sur leur pratique. Dans le cas de l'activité de la SFS, la forme sociale de l'activité est plus difficile à cerner : enseignants formés et formateur pilotent, tour à tour, les échanges. L'opération de reformulation se fait plus rare : les conceptions initiales des enseignants formés ne sont pas (re) mises en question.

\section{Conclusion}

L'originalité de notre démarche est d'examiner les pratiques de formation à l'intérieur des cours de didactique du français pour voir comment la pratique professionnelle y est mobilisée. Des séries de questions ont jalonné ce texte pour nous amener pro- 
gressivement à cerner notre objet d'étude : la circulation des savoirs, liés à l'enseignement de la production écrite en français, entre les temps de formation et les terrains scolaires, pour des étudiants se destinant à l'enseignement primaire et des enseignants en formation en enseignement secondaire. Afin d'observer cette circulation, nous avons choisi de porter notre focale d'analyse sur des activités où les interactions entre formateurs et formés sont centrées sur le retour de la pratique.

La centration sur un objet précis, l'enseignement de la production écrite, met en exergue les contenus et démarches privilégiés et les logiques d'emboitement et d'enchainement que constituent les SF. Nous en avons analysé deux en particulier, la première, sur la conception et mise en place d'une séquence d'enseignement sur un projet de communication ou un genre textuel; la seconde, sur la création d'une séquence pour travailler la poésie à l'école secondaire. L'identification des blocs d'activités portant sur la pratique des deux séquences révèle des relations terrain-institution distinctes selon la filière d'enseignement : au primaire, le temps de terrain est balisé, sous le contrôle du formateur; pour le secondaire, le temps de terrain est diffus et le formateur y a peu de prises, ce qui laisse la place à davantage de conflits, d'une part, entre terrain et institution de formation et, d'autre part, entre formateur et formés.

Qu'est-ce qui caractérise les deux dispositifs d'alternance portant sur l'enseignement de la production écrite? Le terrain sert à tester l'ingénierie didactique, à l'élaborer ou à réfléchir sur celle-ci. Dans les deux cas, le modèle de séquence d'enseignement à concevoir épouse le modèle de la séquence didactique genevoise, avec mise en situation, production initiale, modules et production finale. Ce qui montre à quel point celui-ci est bien intégré dans les pratiques, quand bien même il s'agit d'enseigner une pratique littéraire telle que la poésie.

Dans la séquence pour le primaire, le passage par la pratique permet de reprendre les concepts didactiques vus en cours et de les mobiliser pour l'analyse des expériences positives et négatives vécues lors du stage. Dans la séquence sur le secondaire, le passage par le terrain apporte des nuances et modalise les propositions d'ingénierie faites en formation.

Que peut-on alors retirer de l'analyse des interactions de formation quant à la circulation des savoirs? Apportent-elles un éclairage particulier sur le développement de l'agir enseignant? Des travaux sur l'analyse de verbalisations d'enseignants montrent que les commentaires recueillis auprès d'enseignants novices se répartissent en trois catégories: ils confirment les pratiques, ils les mettent en question ou ils en proposent une évolution pour les modifier (cf. Laurens, 2015). Qu'en est-il de ces trois catégories dans l'analyse des interactions de formation? Les échanges analysés révèlent en dominante une confirmation des pratiques effectives des enseignants, à partir de ce qui a été éprouvé sur le terrain, celui-ci étant posé comme la réalité indépassable sur laquelle viennent se heurter des savoirs et des principes vus en formation, tels que le travail sur la langue et le texte ou la manière d'aborder la poésie. Les pratiques effectives ne sont pas mises en question, elles ne sont pas non plus appelées à évoluer dans les propos des formés. Ce sont plutôt les savoirs vus en formation que ceux-ci questionnent dans les activités de retour sur la pratique. Ce hiatus mis au jour au fil des analyses d'interactions de formation peut être considéré comme zone potentielle de développement.

Au terme de cette étude, nous en venons à proposer deux pistes pour l'ingénierie de formation en vue d'une optimisation de la circulation des savoirs au sein des activités de retour de la pratique, envisagées ici comme propices au développement professionnel des enseignants. Primo, nous recommandons l'élaboration d'outils d'explicitation de la pratique qui permettent, d'une part, de dépasser le stade de la complainte, et d'autre part, d'amener les étudiants à être en mesure de formuler plus précisément les fondements de leur pratique mis en perspective avec à la fois les contenus de formation et leur héritage scolaire. Secundo, un dispositif de formation qui prévoit un moment de retour sur le terrain doit inclure le recueil des pratiques des formés afin de favoriser une mise en dialogue de ses pratiques entre elles et de les situer au regard des contenus abordés en formation.

\section{Références bibliographiques}

Altet, M. (2002). Les styles pédagogiques. Dans J.-C. Ruano-Borbalan, Éduquer et former (p. 353-357). Auxerre Cedex : Éditions Sciences humaines.

Bautier, E. \& Rochex, J.-Y. (2004). Activité conjointe ne signifie pas significations partagées. Dans Raisons Éducatives, $8199-$ 220.

Bourdoncle, R. \& Lessard, C. (2003). Qu'est-ce qu'une formation professionnelle universitaire? Les caractéristiques spécifiques : programmes, modalités et méthodes de formation. Revue Française de Pédagogie, 142, 131-181.

Bronckart, J. P. (2008). Un retour nécessaire sur la question du développement. In M. Brossard \& J. Fijalkow (Éd.), Vygotski et les recherches en éducation et en didactiques (p. 237-250). Bordeaux : Presses Universitaires de Bordeaux.

Chevallard, Y. (1985/1991). La transposition didactique. Grenoble : La Pensée Sauvage. 
Chevallard, Y. (1999). La recherche en didactique et la formation des professeurs : problématiques, concepts, problèmes. IUFM d'Aix-Marseille, École d'été.

[www.aix-mrs.iufm.fr/formations/filieres/mat/fdf/textes/YC_1999_Ecole_ete.doc]

Cicurel, F. (2005). Fiction, interaction, action : quelques jalons pour l'analyse des discours de la classe. Dans Mochet M.-A. \& Barbot M.-J. (dir.) : Plurilinguisme et apprentissages : mélanges Daniel Coste (p. 15-25). Lyon : ENS LSH.

Cicurel, F. (2011). Les interactions dans l'enseignement des langues - Agir professoral et pratiques de classe. Paris : Didier.

Cicurel, F., Bigot, V. (coord.) 2005. Les interactions en classe de langue. Le français dans le monde, recherches et applications, numéro spécial.

Clerc-Georgy, A. \& Ducrey-Monnier, M. (2013). Une mise en abyme propice au développement professionnel dans la formation des futurs enseignants. Dans J.-P. Bernié \& M. Brossard (dir.), Vygotski et l'école; Apports et limites d'un modèle théorique pour penser l'éducation et la formation (p. 281-292). Bordeaux : Presses Universitaires de Bordeaux.

Clot, Y. (2001). Clinique du travail et action sur soi. Dans J.-M. Baudoin \& J. Friedrich (dir..), Théories de l'action et éducation (p. 255-277). Bruxelles : DeBoeck (coll. Raisons Educatives).

Crahay, M. (2006). Un bilan des recherches processus-produit (Carnets des Sciences de l'éducation de I'Université de Genève). Genève : Université de Genève.

Dugal, J.-P. (2003). Le conseil en formation initiale des enseignants. Intérêt et fonction des savoirs didactiques pour le tutorat des professeurs stagiaires en EPS. Thèse de doctorat non publiée. Université P. Sabatier Toulouse III.

Dugal, J.-P. \& Léziart, Y. (2004). La circulation des savoirs entre recherche et formation : I'exemple des concepts didactiques lors d'une action de formation de conseillers pédagogiques. Revue française de Pédagogie, 149, 37-47.

Fillietaz, L. (2013). La mise en circulation des savoirs en formation professionnelle initiale : une approche interactionnelle et multimodale de l'alternance en formation. Dans J.-P. Bernié \& M. Brossard (dir.), Vygotski et l'école; Apports et limites d'un modèle théorique pour penser l'éducation et la formation (p. 293-308). Bordeaux: Presses Universitaires de Bordeaux.

Fillietaz, L. \& Schubauer-Leoni, M.-L. 2008. Les processus interactionnels dans leurs dimensions interpersonnelles, socio-historiques et sémiotiques. Dans Fillietaz L. \& Schubauer-Leoni M.-L. (dir.) : Processus interactionnels et situations éducatives (p. 7-39). Bruxelles: De Boeck.

Gagnon R. 2010. Former à enseigner l'argumentation orale. De l'objet de formation à l'objet enseigné en classe de culture générale. Thèse de doctorat en sciences de l'éducation. Université de Genève.

Gagnon, R. \& Surian, M. (2012). Quels savoirs pour l'enseignement du français dans les plans de formation suisses romands? Repères, 44, 93-115.

Kerbrat-Orecchioni, C. (1990). Les interactions verbales - tome 1. Paris : Armand Colin.

Laurens, V. (2015). «L'auto-confrontation : outil d'observation du développement de l'agir d'enseignants novices, Les cahiers de l'Acedle, 12 (2), 355-381 [http://acedle.org/spip.php?article4429]

Martinand, J.-L. (2006, novembre). Relations entre recherche et expertise dans un laboratoire de didactique curriculaire de sciences et techniques. Conférence présentée au symposium international Formation, Apprentissage et Evaluation en Sciences et Techniques à l'Université, Maroc.

Mayen, P. (1999). Des situations potentielles de développement. Éducation Permanente, 137, 65-86.

Mayen, P. (2002). Le rôle des autres dans le développement de l'expérience. Éducation permanente, 139, p. 65-86.

Mayen, P. (2007). Passer du principe d'alternance à l'usage de l'expérience en situation de travail comme moyen de formation et de professionnalisation. Dans Mehran, F., Vanhulle, S. \& Ronveaux, Ch (dir.)., Alternances en formation (p. 83-100). Bruxellles : De Boeck (Raisons Educatives).

Merhan, F., Ronveaux, Ch. \& Vanhulle, S. (2007). Introduction. Dans F. Merhan, Ch. Ronveaux \& S. Vanhulle, (dir..), Alternance en formation (p. 7-45). Bruxelles : De Boeck (coll. Raisons Éducatives).

Pastré, P. (2005). Dynamique et métamorphose des compétences professionnelles. Psychologie du travail et des organisations, 11, 73-87.

Perrenoud, Ph. (1994). La formation des enseignants entre théorie et pratique. Paris: L'Harmattan.

Portugais, J. (1995). Didactique des mathématiques et formation des enseignants. Berne : Peter Lang.

Prot, B. \& Schneuwly, B. (2013). Introduction et clarification du thème (Développement chez l'enfant-développement chez I'adulte. Formation générale-formation professionnelle des adultes). Dans J.-P. Bernié \& M. Brossard (dir.), Vygotski et l'école; Apports et limites d'un modèle théorique pour penser l'éducation et la formation (p. 275-280). Bordeaux : Presses Universitaires de Bordeaux.

Rabatel, A. (2004). Stratégies d'effacement énonciatif et sur-énonciation dans Le dictionnaire philosophique de Comte-Sponville. Langages, 156, 18-33.

Rossari, C. (1994). Les opérations de reformulation : analyse du processus et des marques dans une perspective contrastive 
français-italien. Berne : Peter Lang.

Schön, D.-A. (1994). Le praticien réflexif. A la recherche du savoir caché dans l'agir professionnel. Montréal : Les Editions Logiques.

Sinclair, J., Coulthard, R. (1975). Towards an Analysis of Discourse. Oxford: Oxford University Press.

Tardif, M., Lessard, C. \& Gauthier, C. (1998). Introduction. Dans M. Tardif, C. Lessard, \& C. Gauthier, Formation des maitres et contextes sociaux (p. 7-70). Paris: Presses universitaires de France.

Tardif, M. \& Ziarko, H. (1997). Introduction. In M. Tardif, \& H. Ziarko (dir.), Continuités et ruptures dans la formation des maitres au Québec (p. 1-8). Québec : Les Presses de I'Université Laval.

Vanhulle, S. (2009). Des savoirs en jeu en savoir en je. Cheminements réflexifs et subjectivation des savoirs chez des jeunes enseignants en formation. Berne/Neuchatel : Peter Lang.

Vanhulle, S. \& Lenoir, Y. (2005). L'état de la recherche au Québec sur la formation à l'enseignement. Vers de nouvelles perspectives en recherche. Montréal : Éditions du CRP, Université de Sherbrooke.

Vanhulle, S., Mottier Lopez, L. \& Deum, M. (2007). La coconstruction de soi et de ses savoirs professionnels comme effet de I'alternance : quels indicateurs? Dans F. Merhan, C. Ronveaux \& S. Vanhulle, Alternances en formation (p. 241-257). Bruxelles : De Boeck (coll. Raisons Éducatives).

Vinatier, I. (2013). Le travail de l'enseignant. Une approche par la didactique professionnelle. Paris : de Boeck. 